\title{
Can one pay attention to a particular color?
}

\author{
PETER BRAWN and ROBERT J. SNOWDEN \\ Cardiff University, Cardiff, Wales
}

\begin{abstract}
In an array of elements whose colors vary can we selectively choose to process all the items of a particular color preferentially in relation to those of another color? We addressed this question by presenting subjects with arrays containing many elements, and recording reaction times to a luminance change of one of the elements. Half the elements had one color and the other half another color-the spatial distribution being random. In two tasks - a simple detection of this change or a choice reaction time to the polarity of the change-we found that reaction times were independent of the number of items in the array. Cuing the subjects as to the color of the target item had no significant influence on the detection task, but subjects were faster if cued for the discrimination task. A further experiment replicated these findings and examined possible costs and benefits. Our final experiment separated the roles of attentional guidance and postattentional processes by having subjects judge the orientation of the target element and varying the magnitude of the target flash that defined which element was the target. We found that this judgment was also affected by color cuing, and that the size of the effect interacted with the flash strength, suggesting that color cuing has its influence at the stage of attentional guidance. We conclude that subjects can selectively attend to items on the basis of color given the appropriate task and stimulus dynamics.
\end{abstract}

In everyday life we are bombarded by a mass of stimulation through our senses. Yet we seem only to be able to process a limited amount of this information. For instance, our highest acuity is available only at the point of foveation, so if we want to examine the fine detail of an object, we must select this object and move our eyes so that its image falls on our foveal retina. One could say that this object had been selected for an overt movement of attention. However, we are also familiar with covert movements of attention. For example, if we hear our name mentioned at a party we might "decide to listen" to the person that had muttered our name without actually moving our external sense organs. One can then ask the question as to how one listens to that particular person. Is it because he/she is standing in a particular position, so the sounds emanating from that position are selected, or is it because of the "sound quality" (such as pitch or timbre) of that person that allows for his/her voice to be selected? In this paper we examine a visual analogue of this situation. Namely, can information from the visual scene be selected on the basis of position, on the basis of some other visual quality (in this case we chose color), or both?

On the question of whether visual stimuli can be selected on the basis of position, the answer from a wealth of previous research is an overwhelming "yes." For example, Posner and colleagues (Posner \& Snyder, 1978; Pos-

P.B. was supported by an EPSRC studentship (B 93314564). We thank the reviewers for their most valuable suggestions, and in particular to Hermann Müller and Jan Theeuwes for criticisms that helped us formulate Experiment 4. Correspondence should be addressed to R. J. Snowden, School of Psychology, Cardiff University, Cardiff CF $13 Y$ G, Wales (e-mail: snowden@cardiff.ac.uk).

-Accepted by previous editor, Myron L. Braunstein ner, Snyder, \& Davidson, 1980) cued subjects as to the likely location of a forthcoming target. They found that subjects were faster if the target was presented at this location than if no cue was given. They also observed that subjects were slower when the target appeared at a location that was different from the cued location than when no cue was given. Thus there appear to be benefits at the attended location and costs at locations from which attention has been withdrawn. Such findings have been repeated on numerous occasions and for many different tasks, such as target detection, target discrimination, target identification (see, e.g., Bashinski \& Bacharach, 1980; Downing, 1988; Hawkins et al., 1990; Hawkins, Shafto, \& Richardson, 1988; Hughes, 1984; Müller \& Humphreys, 1991; Reinitz, 1990; Shaw, 1984). Debate still rages as to the exact nature of the changes that take place under such experimental conditions. For example, some have argued that many results can be explained on the basis of biases in favor of information arising at the cued location (particularly for simple detection tasks, e.g. Shaw, 1984 though there is evidence that this may not be true, Brawn \& Snowden, 1996 , in press). Others have argued that many effects can be explained by the reduction in noise that may be produced by only having to process the target location rather than multiple locations (e.g., Kinchla, 1992; Kinchla, Chen, \& Evert, 1995; Palmer, 1994). Whatever the mechanism of attentional mediation, it appears that there is overwhelming evidence that space can provide a medium through which attention can be modulated. What, then, is the evidence that there may be other media through which attention could be modulated?

\section{Selective Looking}

Some studies have suggested that visual attention to scenes can profoundly alter our perception (Becklen \& 
Cervone, 1983; Neisser \& Becklen, 1975; Stoffregen \& Becklen, 1989). Neisser and Becklen (1975) presented subjects with two movies (one of a hand-clapping game, the other of a basketball game) that were superimposed on each other and hence could not be distinguished by position. The subjects then had to respond to events occurring in one of the movies or in both of the movies. Performance when subjects were attending to one of the movies was very good-as good as if the second had not been superimposed. However, performance was quite poor when the subject had to follow both games. This was not due to the increased rate of events - slowing the movie by a factor of two did not change this result. In further experiments, Neisser and Becklen asked subjects to follow the handclapping game while the researchers presented "bizarre" events in the other game, such as men changing into women. Very few subjects noticed these bizarre events. The conclusion seems to be that subjects have a limited ability to pick up information from both scenes at once. This, at first glance, seems to suggest attention can be mediated through some medium other than space, since the two scenes were completely superimposed. However, there are a number of possible confounds. First, the area of most interest (the movement of the ball for the basketball game, the contact between the hands for the clapping game) would most often be at different spatial locations, so a location-based attentional system that could move with the areas of interest could easily explain this result. Eye movements were also allowed, so covert movements of attention would also be taking place.

Some studies seem less prone to these particular criticisms (Goldstein \& Fink, 1981; Rock \& Gutman, 1981). Rock and Gutman presented subjects with a series of slides on which two simple images were superimposed. One of these images was in green and the other in red. Subjects were informed that they should rate "how pleasant" the red figure was. On a later recognition test, the red figures were well recognized but the green ones were very poorly recognized. Again this seems to highlight the strong effect attention may have; however, one is not sure in this case whether perception or memory has been altered by the experimental manipulations. One might atso doubt that color was the medium through which attention was able to modulate. It seems plausible that the particular object to be attended was marked by its color, allowing a space- or object-based mechanism to move to the appropriate location/object.

More recent studies seem to offer little support to the notion of attentional modulation through color. Shih and Sperling (1996) directly tested this idea using a paradigm that seems to preclude the use of color to signal location. Arrays of elements were presented in rapid succession, and subjects searched for a particular target. Successive arrays could be all of the same color or could alternate between two colors (e.g., red and green). In a series of experiments, they instructed subjects to attend to the green elements only, red elements only, or both. They also pre- sented conditions in which one particular color was replaced by blanks. They found that performance was the same whether the subjects knew the target color or not, suggesting no role for color in modulating attention. In the condition in which arrays of the nontarget color were not shown, performance was better than when they were shown but had to be ignored. This shows that if the other irrelevant colored items could be "mentally" filtered out, performance would be improved. Shih and Sperling went on to show that if the colors provide information about location-which was achieved by having in each array five elements of one color and one odd-colored elementthen considerable attentional effects were demonstrated. Somewhat similar conclusions have been reached through other methods (Laarni, Koski, \& Nyman, 1996). Cave and Pashler (1995) had subjects report the highest digit of a particular color from a string of rapidly presented pairs of digits. Despite these instructions regarding the use of color, they found that subjects made fewer errors when successive targets appeared in the same location than when they switched location. Again this seems to point to the importance of position information over that of color.

Tsal and Lavie (1988) addressed the issue of attention to color through a paradigm that pitted color against location. Subjects were briefly presented with an array of nine letters in a circle. Three of the elements were red, three were green, and three were brown. Subjects were instructed to name a letter of a particular color (let us say "red") and then any other letters. The idea behind this was that if all the red letters became more salient because of the instructions, then it would seem likely that the letters named after the initial one would be more likely to be of this instructed color. If, however, the initial letter dragged attention to this location, then it would seem likely that other letters named would come from nearby locations. The results showed that the additionally reported letters tended to be those near the initial letter and therefore clearly support the location-based view of attention. Taken together, these various studies provide little or no evidence for color-based attentional modulation. Indeed, this position has been made quite explicit: “. . . attention cannot be distributed over a subset of items (e.g., the red ones) when these are spatially scattered among other items in a randomly mixed display" Treisman (1982, p. 199).

The study of Tsal and Lavie (1988) has, however, been reconsidered in the light of possible criticisms. Van der Heijden, Kurvink, de Lange, de Leeuw, and van der Geest (1996) suggested that subjects in the original study may not have remained fixated at the central point but may have shifted their fixation to somewhere on the circle. Wherever their fixation alighted would have been closer to a target element (a red letter) than the fixation point. Hence this shift in fixation may have biased subjects into then selecting the nearby elements, rather than other red ones, because these nearby ones would have been much nearer the fovea, which may be an important consideration for spatial identification tasks due to magnification 
factors (see, e.g., Anstis, 1974). Van der Heijden et al. repeated this experiment with an extra control that the subject had to first identify a digit presented at the point of fixation (in order to ensure correct fixation). They found that red letters were reported three to four times more frequently than nearby ones (Experiment 1). In further experiments (Experiments 5-6), they found that the color advantage was subject to an interaction with visual quality: The poorer the visual quality of the stimuli, the smaller the color advantage. This may well occur because as the visual quality diminishes, subjects are more likely to use the shift of fixation strategy to perform the task. This study suggests that color can be an important mediator in the elements available to the subject.

Another study of interest involves the use of a paradigm introduced by C. W. Eriksen and Hoffman (1972). Here a target element that the subject must identify is flanked by two elements. These flanking elements may be the same as the target element, be neutral (i.e., give no cue as to the correct response), or be different (in that if it were presented as the target element, it would demand a different response than the current target element). Typically such a paradigm shows that speed of response is fastest for same trials and slowest for different trials (B. A. Eriksen \& C. W. Eriksen, 1974). The size of this effect drops off as the flankers are more spatially removed from the target (B. A. Eriksen \& C. W. Eriksen, 1974), demonstrating once again the importance of spatial factors in attention. However, it has also been shown that the effect is greater when the target and flanks share the same color than when they are different (Baylis \& Driver, 1992), and this color effect could even override spatial location in importance.

Of course color is not the only manipulation that may produce cuing effects. For example, knowledge of the likely shape of the target element can also influence the speed of its exact classification (Lambert \& Hockey, 1986).

This brief review has shown that there is some, though limited, evidence of the importance of color in modulating attention. Given its central role in some theories of visual attention (e.g., Bundesen, 1990), it would be of interest to gather further evidence of its existence and role (or lack thereof) in visual processing. To this end we have performed experiments that are analogous to those of the Posner spatial-cuing paradigm except that we have replaced the spatial aspect with a color aspect. Our stimuli consisted of a large number (16-64) of circles that were randomly scattered (but without overlap) on the screen. Half were randomly chosen to be green and half to be red. Subjects were informed of the color of the target in a block of trials (red, green, or either). After a brief period, one of the elements was incremented in luminance and the subject had to respond as quickly as possible to this increment. Such a task is perhaps among the simplest one could devise, but it is known to produce cuing effects in experiments that have used a spatial cue (e.g., Posner et al., 1980).

\section{EXPERIMENT 1}

In this experiment, subjects attempted to respond as quickly as possible to a luminance increment in one of the elements of the display. The color of the element to be incremented was either known or not known, but the location of the element was never known. Simple luminance increments of "large" elements are thought to be detected with similar reaction times across much of the retina, presumably because of the similarity in shape and sensitivity of the underlying temporal channels across the retina (Snowden \& Hess, 1992). This is a useful property because it means that elements at the edge of the display should have similar reactions to those at the center and pilot data in the present setup confirmed this finding. Hence if the subject were to overtly move his/her attention to a particular element (we stress, however, that subjects were instructed not to do so), this overt movement would have had no effect on reaction times. One might argue that the subject might (covertly) pick a particular element and pay attention to that element. If the color of the target element were known, the subject would have a greater chance of being "correct" (in the sense of this being the element that is the target) than if the target color were unknown. This seems a priori an unlikely strategy. Even under the most favorable conditions (16 elements, color known), the subject would only have a 1 in 8 chance of guessing the correct element, which would decrease to a 1 in 64 chance under the worst condition (64 elements, color unknown). Our manipulation of element number, then, serves as a test for such a strategy. If this is the case, any attentional effects should diminish with increasing number of target elements and would show as an interaction between target color and element number variables. Note also that the same argument would apply if the subjects were able to split their attention over a small number of items (see, e.g., Pylyshyn \& Storm, 1988).

\section{Method}

Subjects. Ten subjects were recruited from staff and students at the University of Wales who were either filling course requirements or were paid for their services. All subjects were screened for normal color vision by the Ishihara color test.

Stimuli. The experiments were run via custom-written software driving a Cambridge VSG2.2 graphics generator and displayed on a Mitsubishi Diamond Pro 20X running at a frame rate of $120 \mathrm{~Hz}$. Responses were accepted via the mouse buttons using a clock on the VSG2.2 that could achieve millisecond accuracy. The display elements were circles of either red or green. The luminance of the red and green stimuli were equated to similar perceptual brightness by a method of adjustment in pilot experiments that resulted in the luminances of $4 \mathrm{~cd} / \mathrm{m}^{2}$ for the red elements and $6 \mathrm{~cd} / \mathrm{m}^{2}$ for the green elements. To further guard against subjects using brightness (and therefore possibly salience; see Kaptein, Theeuwes, \& van der Heijden, 1995) rather than color cues in this experiment, we then introduced a random variation of $\pm 10 \%$ into the luminance of each element (for a discussion of this technique, see Snowden, 1998). The "target" consisted of a luminance increment in one of the elements of $50 \%$ of its original luminance. The background luminance of the display was $0.025 \mathrm{~cd} / \mathrm{m}^{2}$. 
From the viewing distance of $57 \mathrm{~cm}$ each element had a diameter of $2^{\circ}$. Elements were randomly assigned positions on the basis of a $10 \times 10$ grid of possible locations within a display area of $29^{\circ} \times 25^{\circ}$. Within an array the color of each element was chosen randomly except that there was always an equal number of each.

Procedure. On each trial the subject was presented with a fixation cross at the center of the screen, which remained on for the duration of the trial. Subjects were told to fixate this point and maintain fixation throughout the trial. After a period of $2,000 \mathrm{msec}$, the elements of the array were displayed for a period randomly chosen between 1,500 and 3,000 msec. At this point the target element was incremented in luminance and remained at this level until the subject responded, or for $2,500 \mathrm{msec}$, and then the screen was blanked. There was an intertrial interval of $2,000 \mathrm{msec}$. Catch trials were exactly the same except that the luminance of the target element was not incremented. Reaction times and responses were collected by the computer. Reaction times less than 100 or greater than $2,500 \mathrm{msec}$ were discarded.

In each block of trials the subject was informed about the color of the target. There were three blocks corresponding to red targets, green targets, or either red or green targets. The ordering of these conditions was approximately counterbalanced across subjects. In each block of trials, three sets of number of elements $(16,32,64)$ were randomly interleaved.

Each subject completed two blocks consisting of 30 trials each for each of the three main conditions, making a grand total of 180 trials per subject. Catch trials were included, and the ratio of their occur-
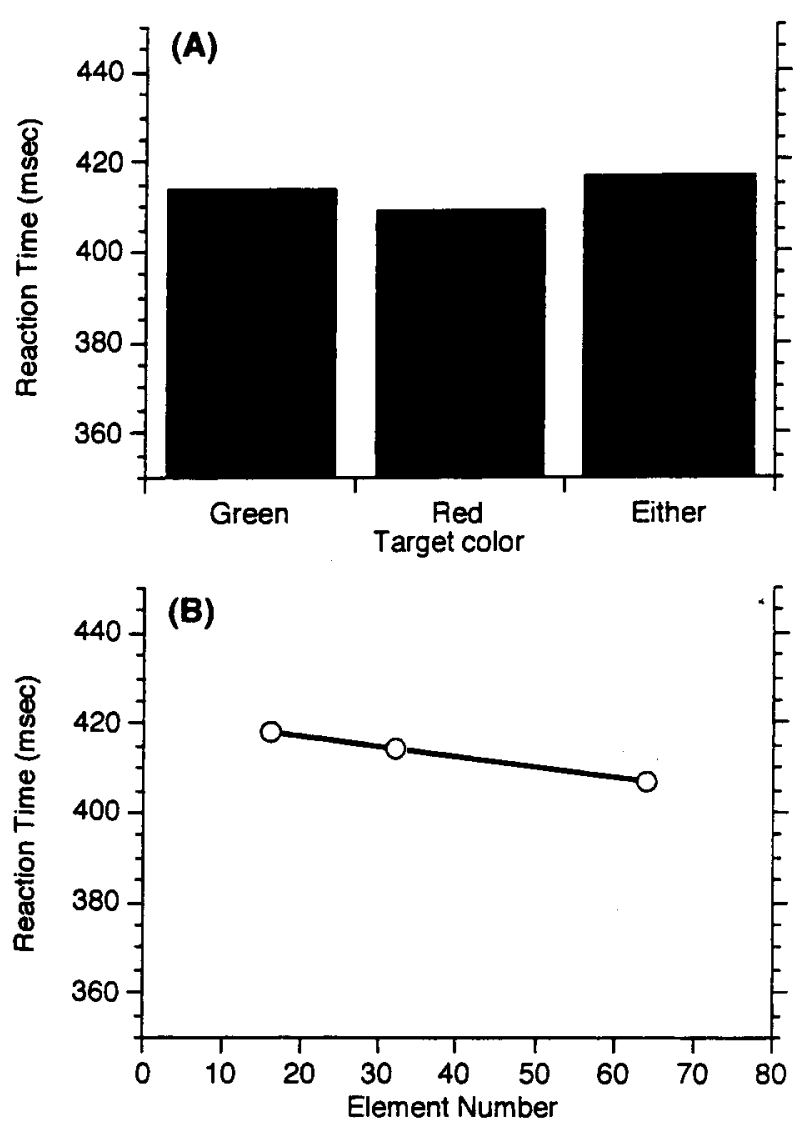

Figure 1. Mean reaction times are presented for the threetarget condition (A) and as a function of element number $(B)$ for the detection of a luminance change in Experiment 1. rence to target present trials was $1: 10$. Breaks were offered to subjects between blocks.

\section{Results and Discussion}

Mean reaction times for target-present trials were calculated for each condition and number of elements for each subject. These mean reaction times were subjected to a repeated measures analysis of variance (ANOVA) with target color (red, green, either) and element number (16, $32,64)$ as factors. The analysis revealed no significant effects of target color $(F>1.0)$ or element number $[F(2,9)=$ $2.14, p>.10]$ or any interaction $[F(2,9)=1.33, p>.10]$. The mean reaction times for target color collapsed across element number are shown in Figure 1A; those for target number collapsed across target color are shown in Figure $1 \mathrm{~B}$.

We also obtained data for misses (when the subject did not respond to the luminance increment) and both correct rejections and false alarms on the catch trials. The percentage of targets missed was $19.5 \%$ when the target color was known and $23.8 \%$ when it was unknown. The percentage of false alarms was $13.3 \%$ when the color was known and $14.8 \%$ when it was not. Due to the small overall number of these instances, we do not present the formal analysis of these data (though the ANOVA revealed that all main effects and interactions were insignificant $[F<1.0])$. The error rates may seem quite high relative to many other cuing experiments. However, one must note that our targets were detectable only due to their "transients." The sustained luminance level was not a good indicator of target presence, since the luminance of each element was randomized (see Method section).

The results of our first experiment, then, offer no support to the notion that subjects can selectively attend to elements of one particular color from within an array of elements containing two colors.

One might argue that this result is not at all surprising in light of the results from the manipulation of target number. Inspection of Figure $1 \mathrm{~B}$ shows that target number had little effect on reaction times (if there is any trend, it is for reaction times to decrease with element number!); this is often seen as the hallmark of a preattentive process (Treisman \& Gelade, 1980). Now let us imagine that we can perfectly pay attention to the target color (to the extent that the other colored elements no longer have any neural representation at an appropriate stage). Reaction times would now mirror conditions in which only half the elements had been presented, but since there is no effect of element number, there will be no change in reaction time! Indeed, this line of reasoning could be applied to all tasks that show flat reaction times versus element number search slopes. Hence one might argue that we have chosen a task in which it should be impossible to get any effects of attention. However, it is clear from experiments using luminance increment detection that many have reported significant effects of spatial attention (see, e.g., Bashinski \& Bacharach, 1980; Downing, 1988; Hawkins et al., 1990; Hawkins et al., 1988; Hughes, 1984; Müller \& Humphreys, 
1991; for counterexamples, see Bonnel, Stein, \& Bertucci, 1992; Shaw, 1984).

Given the doubt concerning whether one should expect any effects of attention on luminance increment detection, one cannot use this task as strong evidence against attention being modulated by color. We therefore sought to examine the issue further through a task that is generally thought to be influenced by attention-luminance change discrimination (Bonnel et al., 1992).

\section{EXPERIMENT 2}

Bonnel et al. (1992) examined subjects' ability to detect the presence of luminance changes in two diodes, one on either side of the fixation point. They found no tradeoff with attentional instructions and therefore suggested that luminance change detection was not altered by attention. However, if the subject had to perform luminance discrimination (i.e., was the change to darker or brighter?) then cuing effects were obtained. This is a particularly striking result since the stimuli for the two experiments were very similar (though the duration of the changes did vary in order to give comparable performance); thus it was only the task of the subject that influenced whether attentional effects were obtained (for a similar argument regarding detection and identification, see Bravo \& Nakayama, 1992). Why is this the case? One argument is that discrimination tasks require focal attention, whereas detection tasks do not. For example, Sagi and Julesz (1985) have shown that a small number of targets differing in orientation from their background can be detected in parallel, whereas the task of comparing their orientations seems to require a serial process. Hence our task of detection may not have required any shifts of attention. We therefore repeated Experiment 1 except that the luminance changes could now be either increments or decrements and the subjects' task was a choice reaction time as to the polarity of this change.

\section{Method}

Subjects. Ten subjects were recruited for this experiment under the same conditions as in Experiment 1.

Stimuli. The stimuli were the same as in Experiment 1 except that the target luminance change was $\pm 50 \%$. The polarity of this change was chosen randomly for each trial. No catch trials were included because this was a choice reaction time.

Procedure. The procedure was the same as in Experiment 1, except that the subject now had to make a choice reaction time by pressing one button for luminance increments and the other for decrements.

\section{Results and Discussion}

Mean reaction times for target-present trials were calculated for each condition and number of elements for each subject. These mean reaction times were subjected to a repeated measures ANOVA with target color (red, green, either) and element number $(16,32,64)$ as factors. The analysis revealed a significant effect of target color $[F(2,9)=7.90, p<.005]$ but not element number $(F<$
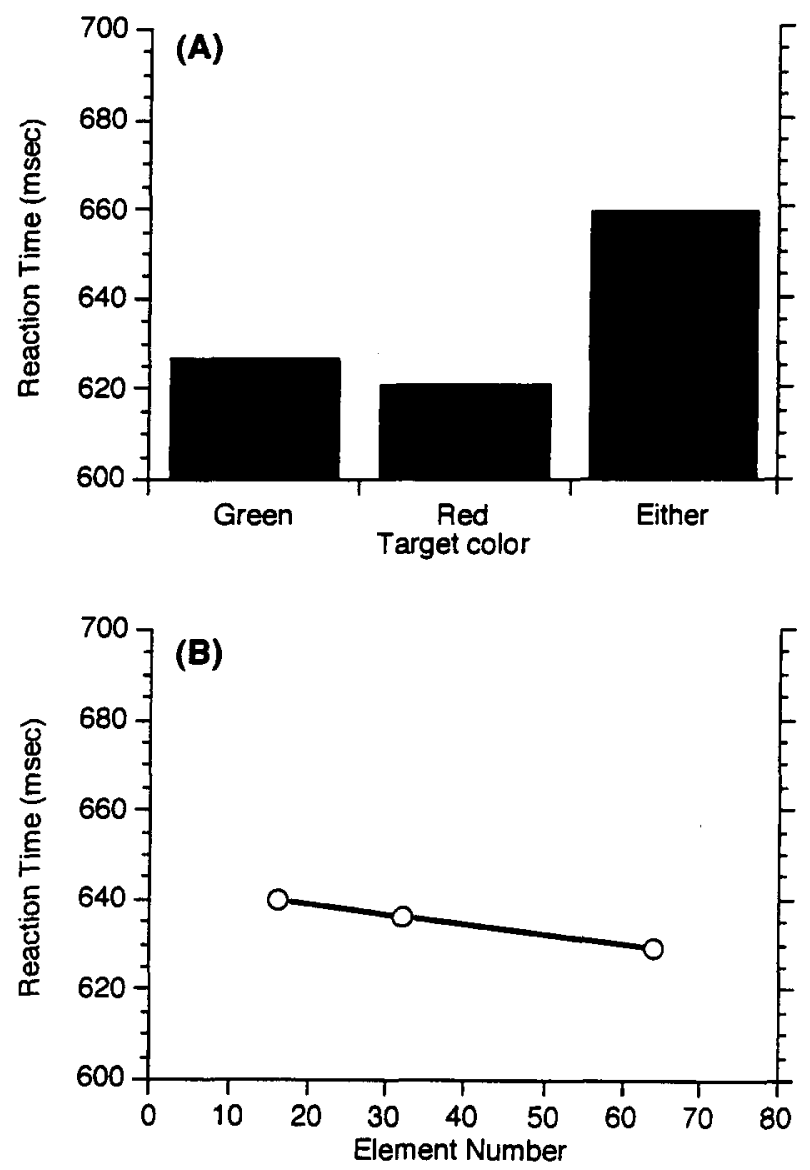

Figure 2. Mean reaction times are presented for the threetarget condition (A) and as a function of element number $(B)$ for the discrimination of the polarity of a luminance change in Experiment 2.

1.0), nor was there a significant interaction $[F(2,9)=1.36$, $p>.10]$. The percentage of targets missed was $9.5 \%$ when the target color was known and $10.5 \%$ when it was unknown (note that, as before, although the targets remained at the new luminance value, they were defined as targets only by the transient part of the luminance change because of the randomization in each element's luminance-hence it was still possible to have misses). In an ANOVA this effect was insignificant $(F<1.0)$. The percentage of targets correctly discriminated was $96.9 \%$ when the target color was known and $96.1 \%$ when it was not. Again, this effect was shown to be insignificant when subjected to an ANOVA. The mean reaction times for target color collapsed across element number are shown in Figure 2A, and for target number collapsed across target color in Figure 2B. Inspection of these figures shows that subjects were indeed slower in discriminating polarity changes when they could occur in either the red or the green elements than when the color of the target elements was known.

Once again in this experiment we did not obtain an increase in reaction time as the number of elements increased. This leads to a problem in explaining the atten- 
tional effects here in terms of "filtering out" (Broadbent, 1958 ) items that do not have the target color. If elements that are not in the target color (in this case, half the elements) could be prevented from further processing at a very early stage, then the results should resemble those found for searches with fewer elements. However, we see that altering element number has no effect (or if the trend in the data is to be believed, then reaction times should increase with decreasing element number). Hence the decrease in reaction time caused by color cuing does not resemble the results one would expect if the representation of the elements with the nontarget color were eliminated early in processing. This apparent lack of filtering out of the nontarget items is in line with the findings of Shih and Sperling (1996), described in the introduction.

The lack of interaction of the attentional effect with element number is also worth noting. As described earlier, one might imagine an explanation of the present results in terms of the subject "guessing" which element(s) to pay attention to -a guess that would be more likely to be correct if the color of the target element is known. Such a strategy should be less effective as the number of elements increases (as the chance of guessing correctly decreases). To further examine this issue, we tested the effects of attention at each set size separately. First, planned comparisons between the three conditions revealed significant effects at each set size (all $p \mathrm{~s}<.05$ ). Second, the size of the effect (calculated by either - [red + green $] / 2$ ) was $28.6 \mathrm{msec}$ at $16,45.8 \mathrm{msec}$ at 32 , and $30.8 \mathrm{msec}$ at $64 \mathrm{msec}$. None of these data indicate a trend toward the effect lessening at higher set sizes; the data argue against the guessing strategy as an explanation of the attentional effects reported here.

\section{EXPERIMENT 3}

The results of the previous two experiments suggest that attention can be modulated by color in a task that requires focal attention (luminance discrimination), but not when the task does not require focal attention (luminance detection). As mentioned earlier, there have been many studies in the domain of spatial attention, some of which have produced attentional effects and others of which have not. We were therefore concerned that our conditions may not have been ideal for the detection of attentional effects. One concern is that the trials for a particular target color condition were presented in a block. Such a blocking of endogenous attention cues has been shown to considerably reduce attentional effects (Posner et al., 1980). Therefore, in Experiment 3 the cue to the target color (red, green, or either) was presented briefly at the beginning of each trial. Given that these concerns led us to attempt replications of Experiments 1 and 2 under these nonblocked conditions, we took advantage of this opportunity to improve the design and investigate the nature of the attentional modulation (if it still existed!). The small improvement was that in Experiment 1 the luminance changes were all increments, whereas in Experiment 2 there were both increments and decrements. Although we could not see any theoretical reason why this should change attentional process or strategies, we rectified this anomaly for Experiment 3 by presenting both increments and decrements in both the detection and the discrimination experiments.

We also changed our paradigm so that we could look at both the possible costs and the possible benefits of attention. In Experiments 1 and 2 we compared cued conditions (red or green) with neutral conditions (either) and so only examined the benefits that could accrue from attention being on one color versus spread between both colors. In Experiment 3 we used Posner et al.'s (1980) technique in occasionally presenting "invalidly" cued trials (e.g., cuing red but then the target was a green element) that would allow us to examine possible costs of attention as well as its benefits.

\section{Method}

Subjects. Fourteen subjects were recruited for this experiment under the same conditions as in Experiment 1. The data of 1 subject, however, were excluded from analysis when during debriefing he claimed to have made no attempt to follow the cues.
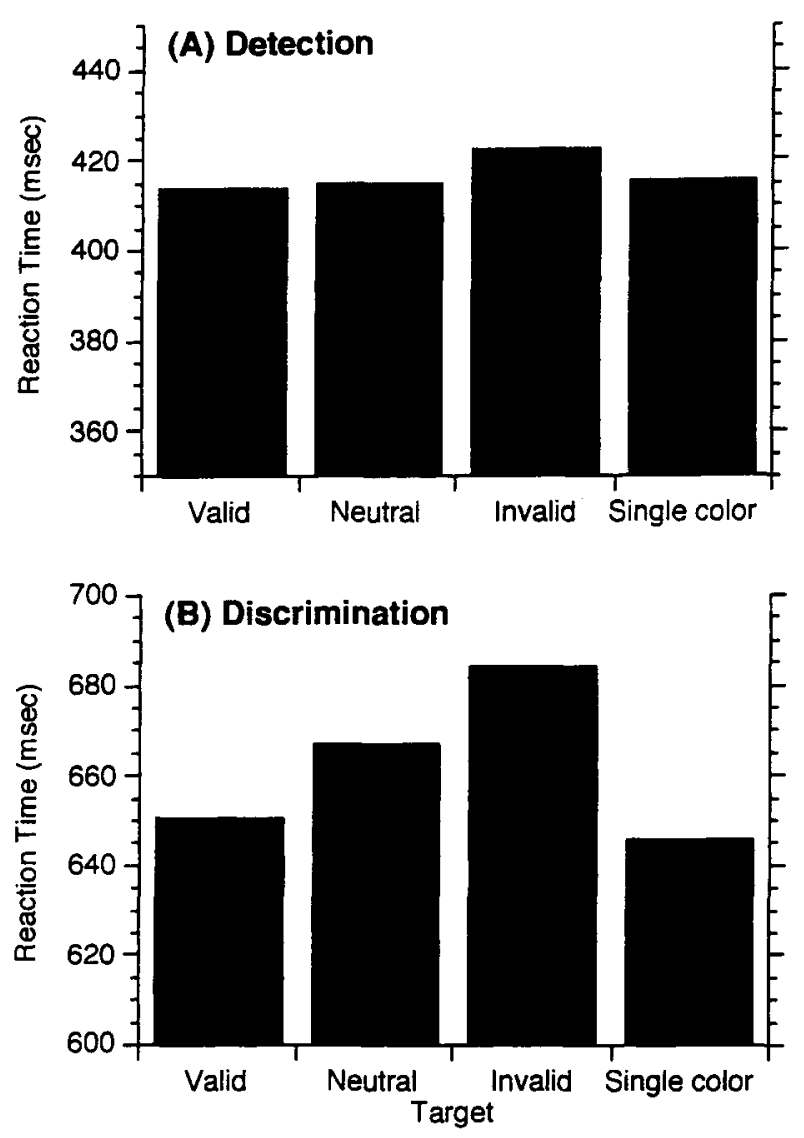

Figure 3. Mean reaction times are presented for the fourtarget conditions in Experiment 3 for the detection of a luminance change (A) and for the discrimination of the polarity of a luminance change $(B)$. 
Stimuli. The stimuli were the same as in Experiment 2 except that in this experiment, 32 elements ( 16 red, 16 green) were presented in Conditions $1-3$. For Condition 4 , only 16 elements were presented, all of one color. The cuing of the target element color (see Procedure section) was achieved by presenting a small circle of $8^{\circ}$ diameter that was displayed until the subject instigated the start of the trial. This cue was the appropriate color for valid trials and for the single-color condition, was of the wrong color for invalid trials, and was white for neutral trials.

Procedure. The procedure was similar to that in Experiment 1 for the detection task. The major change was in the validity of the cue. Within a block of 250 trials, four conditions were randomly interleaved. In Condition 1 (valid), a color cue indicated which set of colored elements would contain the target and was correct; 100 trials $(40 \%)$ were of this type. In Condition 2 (invalid), a color cue indicated which set of colored elements would contain the target and was incorrect; 25 trials $(10 \%)$ were of this type. In Condition 3 (neutral), a white cue indicated either set of colored elements was equally likely to contain the target; 50 trials $(20 \%)$ were of this type. In Condition 4 (single color), a color cue indicated the color of all 16 elements and was therefore always correct; 25 trials (10\%) were of this type. Finally, 50 catch trials $(20 \%)$ were included that were spread across all conditions so that the chance of a catch trial was equally likely on any trial. The discrimination task followed similar procedures as the detection task except that the subject made a choice reaction time as to the polarity or the luminance change and except that there were no catch trials. Detection and discrimination tasks were run in separate blocks, and the ordering of the two blocks was randomized from subject to subject.

\section{Results}

Detection experiment. Mean reaction times for the four conditions in the luminance detection experiment are plotted in Figure 3A. Inspection of this figure shows little effect of condition on reaction time. A one-way ANOVA showed no significant effect $[F(3,12)=1.34, p>.10]$. A planned comparison between the valid and invalid trials was also insignificant $(F<1.0)$. The number of targets missed was also examined. Here, too, no significant differences were found $[F(3,12)=1.02, p>.10]$.

Discrimination experiment. Mean reaction times for the four conditions in the luminance discrimination experiment are plotted in Figure 3B. Inspection of this figure shows some effect of condition on reaction time such that the fastest reaction times occurred in the valid and single-color conditions and the slowest occurred in the invalid condition. A one-way ANOVA showed a significant effect of condition $[F(3,12)=4.28, p<.05]$. Planned comparisons showed that the difference between the valid and invalid trials was significant $[F(1,12)=9.79, p<$ $.01]$ but that the difference between the valid and neutral conditions was not $[F(1,12)=1.17, p>.10]$, and neither was that between the neutral and invalid trials $[F(1,12)=$ $2.96, p>.05]$. Once again, the number of targets missed was examined and found to be nonsignificant $(F<1.0)$, as was the percentage of targets correctly discriminated $[F(3,12)=1.19, p>.30]$.

\section{Discussion}

The results of Experiment 3 are in general agreement with those of Experiments 1 and 2. We obtained no significant differences between the various conditions when the task was that of merely detecting the presence of a luminance change, whereas we found significant effects when the task was changed to that of discriminating the polarity of this change. There are, however, some small differences between the results of Experiment 3 and those of Experiment 2. In Experiment 2, we obtained a difference of approximately $35 \mathrm{msec}$ between color-known versus color-unknown conditions (which was statistically significant), whereas the most similar conditions of Experiment 3 (valid vs. neutral) produced a difference of around $17 \mathrm{msec}$ (which was not statistically significant). This should be expected since the cue was $100 \%$ valid in Experiment 2 but only $80 \%$ valid in Experiment 3, and hence subjects may have given it less weight.

A second aim of Experiment 3 was to assess if the improvement in performance (benefit) when the subject is aware versus unaware of the target color is mirrored by a detriment (cost) if this cue is incorrect. Although the data are suggestive that there are both benefits and costs, and that they are approximately equal under the present conditions, both of these trends failed to reach statistical significance.

\section{DISCUSSION OF EXPERIMENTS 1-3}

Our results suggest that cuing target color improves performance in discrimination tasks but not in detection tasks. In the General Discussion we will examine alternative accounts of these data. However, it is germane at this point to give a brief theoretical account of how the data might arise so that our reasons for the next experiments will be clear. We believe that our data are most consonant with a class of models developed by Wolfe and colleagues and given the general term guided search (see, e.g., Wolfe, 1994). These models suggest how bottom-up activation can be supplemented by top-down knowledge to produce an "activation map" of space through which focal attention is guided. Thus tasks that require focal attention are speeded by target color cuing, whereas those that do not require attention are unchanged. We suggest that target discrimination does indeed require focal attention, and there is much evidence from other paradigms to support this notion (see also, e.g., Sagi \& Julesz, 1985). On the other hand, it is possible that the mere detection of the presence of a target does not (see also, e.g., Sagi \& Julesz, 1985). This could be explained on two accounts. First, the response in detection tasks does not require that the subject know about the identity of the target, but merely that it has occurred. This could be signaled by activity at the level of the bottom-up maps and thus without recourse to any master activation map. Since we are postulating that knowledge of the stimulus attributes has its effect at the level of the master activation map, this explains why we see no effect of this knowledge for detection tasks; we shall term this the "no-MAP guidance theory." A second possibility is that the response could still be based on the master activation map but that the top-down modulation is much reduced in this task so that it is no longer seen in 
such experiments; we shall term this the "MAP guidance theory." Why would there be less modulation? Perhaps the subjects found this task much easier than the discrimination task and therefore did not follow the cues as vigorously as in a discrimination task, or perhaps the mere detection of a stimulus requires far less focusing of attention at the correct location. Note that these two theories have many similarities.

An alternative explanation is that knowledge of the target color acts after attention has been attracted to the target element. Processing of targets of the correct color might then proceed more rapidly than those of the incorrect color. Such a model easily explains why the discrimination task is improved by color cuing and can explain the lack (or at least relative lack) or effect on detection tasks by the very reasonable assumption that detection does not require much further processing. We shall term this the "postguidance theory."

Thus we have a number of possible explanations of the data presented so far. The next experiment attempts to address these ideas. The experiments also have the secondary aim of generalizing the results. So far we have examined only decisions about the polarity of a luminance change; the next experiments require the subject to identify the orientation of the target element.

\section{EXPERIMENT 4}

The major distinction between the theories outlined above is in the site of the effects of color knowledge (or attention to color). The first two theories (no-MAP and MAP) suggest that it has its effect on guiding focal attention to the correct element, which is then processed "normally." The latter theory (postguidance) suggests that guidance to this element is not influenced by color cuing but that the processing of the element is slowed once attention arrives there if the element is the incorrect color (or, alternatively, is speeded if it is the correct color). Therefore, in order to distinguish between these theories, we must separate what attracts attention to the appropriate

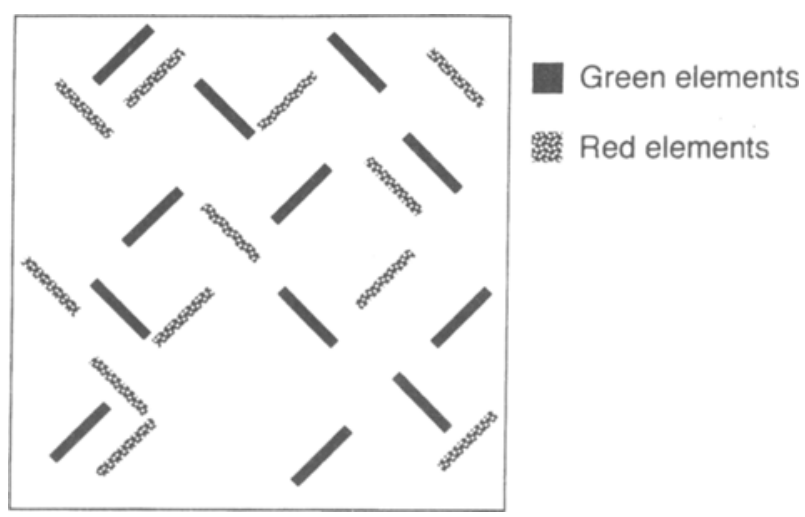

Figure 4. Stylistic representation of the stimuli used in Experiment 4. element from the processing that is required once attention arrives at this location. In Experiment 4, we concentrated on the first part of this process by manipulating the "attention-attracting" properties of the target. We make the reasonable assumption that weak flashes will be less attention attracting than strong flashes. However, the flash is not what the subject must judge; it merely tells them which element to judge. The judgment to be made is now that of the orientation of the target element (Figure 4). Note that this experiment appears superficially similar to those performed by Hughes (1984) and by Hawkins et al. (1988), who varied target luminance in a spatial-cuing paradigm. However, our study differs in a very important way. In these earlier studies, the magnitude of the luminance flash that attracted attention (i.e., the onset of the stimulus) would always covary with the overall brightness of the stimulus; thus this variation in intensity might be expected to have repercussions for both the attentional guidance stage and for postattentional processes. However, in our paradigm the flash alters the luminance of the target only a little from its initial high value, and does so in either direction (both increments and decrements occur in equal number). So we should expect that variations in magnitude should have little consequence for postattentional processes but considerable impact on the attraction of attention to this element.

Let us first consider the discrimination task that all theories agree requires guidance to the element and the further processes of focal attention. Thus reducing the salience of the flash should slow the guidance of attention to the correct element, and this would increase reaction times for target discrimination. If the effect of cuing is indeed at the stage of helping to guide attention to the appropriate element (the no-MAP and MAP theories), we should expect to see an interaction between the cuing condition and the strength of the flash (Sternberg, 1969). However, if color cuing alters the processing after the engagement of focal attention (the postguidance theory), then, whereas changes in flash strength will slow attention arriving at this element, the task to be performed once attention gets there will be unchanged and there should be no interaction. Thus the no-MAP and MAP theories predict an interaction whereby there will be greater effects of color knowledge for low flash strengths, whereas the postguidance theory predicts similar effects of cuing at both flash strengths.

Let us now consider the detection task. The no-MAP theory suggests that the detection response is generated without recourse to the master activation map (which guides attention), and hence there should be no interaction between the cuing condition and the flash strength. The postguidance theory places the effects of cue condition after the attentional guidance and hence again predicts no interaction between these variables. However, the MAP theory does require the attentional guidance of the master activation map. It was suggested earlier that we did not see its effects for the detection task because the task was too easy and did not require precise localization of the 
(A) Detection

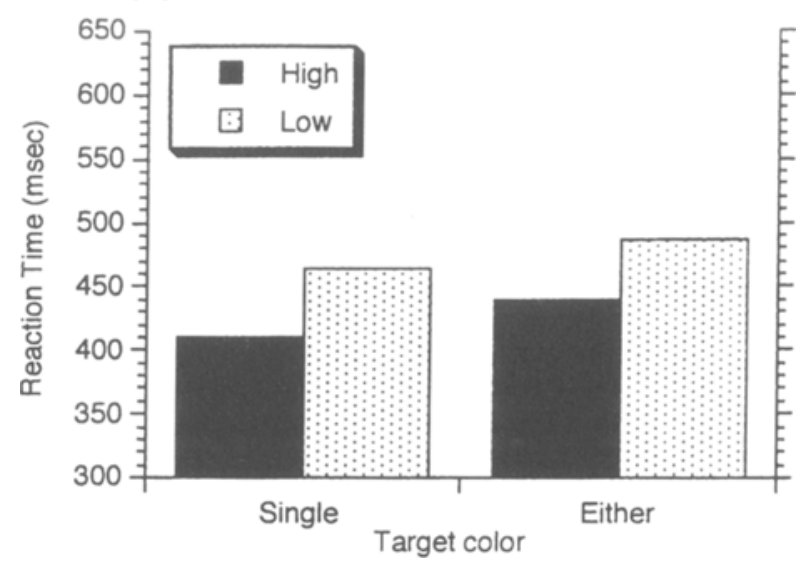

(B) Discrimination

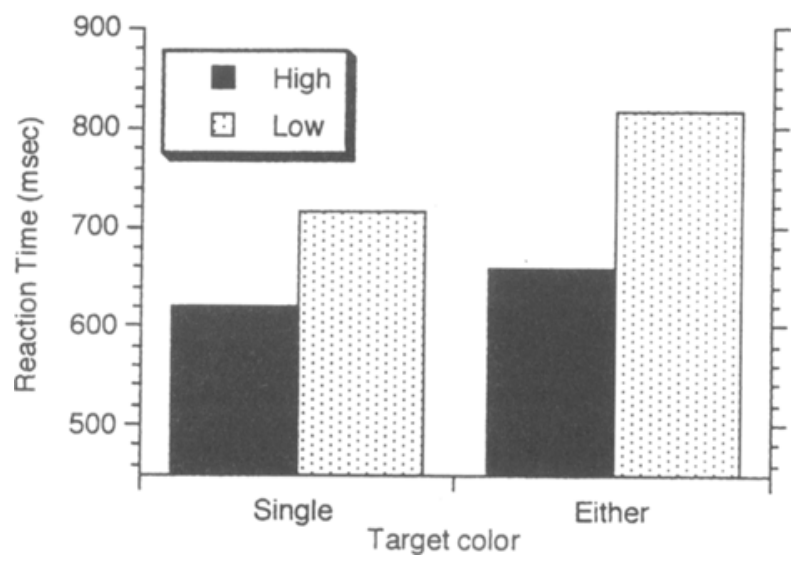

Figure 5. Mean reaction times are presented for the fourtarget conditions in Experiment 4 for the detection of a luminance change (A) and for the discrimination of the orientation of the element that underwent a luminance change $(B)$. The strength of the flash that signaled the target element could be either high (75\% —darker bars) or low (30\%-lighter bars).

signal. One would therefore expect that making the detection more difficult (reducing its luminance) might reveal the effects of attentional guidance. Hence this theory predicts an interaction with a greater effect of color cuing at the low flash strengths.

\section{Method}

Subjects. Ten subjects were recruited for this experiment under the same conditions as in Experiment 1.

Stimuli. The stimuli consisted of 24 elements (12 red, 12 green) arranged under the same rules as in Experiment 1. Each element was a rectangle of $0.5^{\circ}$ width and $2^{\circ}$ height. Half the elements were tilted $45^{\circ}$ clockwise and the other half were tilted $45^{\circ}$ counterclockwise, with no relationship between element tilt and element color (Figure 4). Target flashes were now of either $75 \%$ (high) or $30 \%$ (low) and could be of either polarity (randomly chosen from trial to trial). All other aspects were the same as in the earlier experiments (including the randomization of initial luminance).
Procedure. We returned to cuing the subjects at the start of each block as to the color of the target elements within the block (as in Experiments 1 and 2). Where possible, the procedures and stimulus timings were similar to those in Experiment 1 for the detection task and to those in Experiment 2 for the discrimination task. For both tasks, the number of elements on the screen was always 24 . For the detection task, the subject made a simple reaction time response by pressing a single button. For the discrimination task, the subject made a choice reaction time response as to the orientation of the target element by pressing one of two buttons. Each subject completed two blocks of the three main conditions (cue green, cue red, and no cue) with the order of blocks being randomized both within and between subjects. Within a block of trials, the two levels of target flash were randomly interleaved ( 30 trials of low flash strength and 30 of high flash strength). Thus subjects completed a total of 360 target trials for the detection task and 360 for the discrimination task. For the detection task, we also included catch trials that occurred with a probability of .1 . Thus the experiment had two manipulations, color cuing condition (red, green, either) and flash strength (high, low).

\section{Results}

Initial analysis of the results showed no differences between the cue-red and cue-green conditions for either task. We therefore simply took the mean of these two measures for further analysis.

Detection experiment. Mean reaction times for the four conditions in the luminance detection experiment are plotted in Figure 5. A two-way ANOVA showed a significant effect of flash strength $[F(1,9)=70.73, p<.0001]$, but no reliable effect of cuing $[F(1,9)=2.46, p>.10]$. Most important was the lack of any sign of an interaction between these variables $(F<1.0)$. Analysis of miss rates revealed a similar pattern of results (Table 1). There was a significant effect of flash strength $[F(1,9)=112.66, p<$ $.0001]$, but no reliable effect of cuing $[F(1,9)=3.67, p>$ $.05]$ and no interaction between these variables $(F<1.0)$. It is notable that for both the reaction time data and the misses, the trend in the data concerning color cuing is toward a small effect (subjects were faster and missed fewer of the targets). However, the false alarms were also higher in the single-color condition $(27.7 \%)$ than in the eithercolor condition (16.2\%), suggesting that this trend may reflect a speed-accuracy tradeoff.

Table 1

Misses, False Alarms (FAs), and Error Rates for Experiment 4

\begin{tabular}{ccccc}
\hline \multicolumn{1}{c}{ Task } & Flash Strength & Misses & FAs & Errors \\
\hline Detection & & & & \\
Single & low & 36.4 & 27.7 & - \\
& high & 13.9 & - & - \\
Either & low & 40.4 & 16.2 & - \\
& high & 20.6 & - & - \\
Discrimination & & & & \\
Single & low & 35.2 & 9.5 & 7.8 \\
& high & 11.9 & - & 6.3 \\
Either & low & 38.6 & 8.7 & 14.9 \\
& high & 10.5 & - & 3.9 \\
\hline
\end{tabular}

Note-For the false alarms there is no distinction between high and low flash strengths because no flash is presented. 
Discrimination experiment. Mean reaction times for the four conditions in the orientation discrimination experiment are also plotted in Figure 5. A two-way ANOVA showed a significant effect of flash strength $[F(1,9)=$ 43.22, $p<.001]$, a significant effect of cuing $[F(1,9)=$ $5.87, p<.05$ ], and a significant interaction between these variables $[F(1,9)=5.14, p<.05]$. This interaction consists of a greater cuing effect for the low flash strength trials $(M=99.9 \mathrm{msec})$ than for the high flash strength $(M=$ $39.3 \mathrm{msec}$ ). The data for misses, false alarms, and errors are given in Table 1. Analysis of miss rates revealed a significant effect of flash strength $[F(1,9)=139.36, p<$ $.0001]$, but no reliable effect of cuing $(F<1.0)$ and no interaction between these variables $[F(1,9)=2.22, p>.1]$. Also of interest in this experiment is the error rate (Table 1 ). The ANOVA showed a significant effect of flash strength $[F(1,9)=18.08, p<.01]$, no significant effect of cuing $[F(1,9)=3.13, p>.05]$, but a significant interaction between these variables $[F(1,9)=35.33, p<.001]$.

\section{Discussion}

The results for the discrimination of orientation task showed an interaction between flash strength for both reaction times and accuracy. This is the pattern of results predicted by models that place the effects of color cuing at the guidance mechanisms for attentional focus and seems to speak strongly against theories that place the effect of cuing beyond the guidance of attention. The results also help to generalize the finding of the effects of color cuing to tasks other than polarity judgments. The lack of interaction for the detection task may help discriminate between the two versions of the theories that do place the effects of color cuing on guidance mechanisms. The noMAP theory suggests that the master activation map is not needed (and therefore not used) in such tasks; hence no interaction is predicted. The MAP theory suggests that the master activation map is needed but that the top-down effects of color may not be operating in tasks that are not demanding enough. Hence this theory predicts that effects might be seen for very demanding detection tasks. We did not observe such effects here when the detection task was made more difficult. However, this distinction is now based on a failure to reject a null hypothesis and should be taken with caution. One might argue that our manipulation of flash strength was still not enough to demand the attentional processes of our subjects. We would, however, point out the effects of this manipulation were highly significant and that the subjects appeared to have found the task very difficult, completely missing $38.4 \%$ of the low flash strength targets.

One might again argue that the differences (whether qualitative or quantitative) between the detection and discrimination task might reflect that subjects were "trying harder" to follow the cue for the discrimination task, and hence more attentional effects were observed. If this were the case, we would expect similar patterns of results for all our measurements in the discrimination task. However, it is notable that the misses show a very different pattern of results from that of the accuracy and reaction time data. For the misses, we get a large effect of flash strength; not surprisingly, subjects missed more of the weaker targets. However, we found no effect of color cuing nor any interaction - the same pattern of results as shown for the detection task! This is actually to be expected since misses are indeed a measure of detection rather than discrimination. Thus it appears that the effects of color cuing, and their interaction with flash strength, are dependent on the type of decision the subject is making, rather than any artifact of attentional effort between the two tasks.

\section{GENERAL DISCUSSION}

We have investigated whether subjects can selectively pay attention to all elements of a particular color within a display. To investigate this, we presented subjects with a display containing numerous elements, half of which were one color and half of another color. After a period of time, the luminance of one of the elements was altered and subjects responded as quickly as possible to this change. We found that color cuing did not influence (or had little influence on) the detection of this change, but did improve the subject's ability to discriminate the polarity of the change or the orientation of element that underwent the change.

\section{Guided Search}

Our displays, though designed to be a "color" equivalent of a spatial cuing paradigm, in many ways resemble those used in visual search paradigms. Typically in these paradigms a target is predefined by the experimenter (e.g., a red vertical line) and the subject's task is to report on the presence or absence of this target. Search for a target defined by a single feature (e.g., a red vertical line in a field of green vertical lines) is normally independent of the set size, whereas search time for a conjunction of features (e.g., a red vertical line in a field of red horizontal lines and green vertical lines) often increases with increasing set size. The present experiments, though involving quite different temporal dynamics to more traditional visual search experiments, can be thought of as feature searches in that subjects merely have to detect the single change in luminance. In line with this we found no effect of set size. Is there evidence that performance in other feature searches can be affected by color knowledge? Although there are many examples of how color may help in cases where subjects must overtly or covertly scan complex (feature conjunction) targets-that is, those that show the hallmarks of serial search (see, e.g., Carter, 1982; Egeth, Virzi, \& Garbart, 1984; Green \& Anderson, 1956; Kaptein et al., 1995; Laarni et al., 1996; Smith, 1962)-there is little evidence that bears on the issue of single-feature searches (i.e., those that show the hallmarks of parallel search). An example would be for searches for a red vertical line in a field of red horizontal and green vertical lines. Though some experiments appear to have had subjects searching for a such a target, they either have had no control condition where the elements are all the same color (Friedman- 
Hill \& Wolfe, 1995, Experiment 1) or the subject was unaware of which color would be the target from trial to trial (Snowden, 1998). One experiment does seem to have the correct conditions. Treisman (1988) measured search for a horizontal line in a field of vertical lines. The target was always green and the distractors were either all green (homogeneous control) or of three colors. No significant results were found in this experiment. The present experiments therefore seem to be the first to report that a single-feature search (in this case for a "dynamic" feature) can be aided by knowledge of another feature that the target will have (even though knowledge of this feature gives no indication of the response to be made). The reasons why we found an effect and Treisman did not are not yet clear. There are many differences between the displays that merit investigation, not least the temporal aspects of the targets, and we hope to report on this at a later date.

However, under some conditions feature-conjunction searches are independent of the number of distractors (Egeth et al., 1984; Friedman-Hill \& Wolfe, 1995; Nakayama \& Silverman, 1986; Sagi, 1988). To explain this type of behavior, the notion of guided search has been proposed (for its most recent incarnation, see Wolfe, 1994). These models postulate that feature maps are constructed for the various attributes of the pattern elements. Within these feature maps, interactions take place that define the salience of the various elements, and these various feature salience maps are used to construct a master activation map by summing activity across all such feature maps. These bottom-up processes can be complemented by top-down knowledge - such as knowing the color of the target- to give greater weight to the salience map in the known feature. This master activation map then is used to direct focal attention in order of decreasing activation. It is assumed (by the present authors) that this deployment of focal attention allows the features at this location to become available to a decision-making level, and the response ("target present" or "check next item") is generated. Thus in our example of searching for the vertical red element the "red" map and the "vertical" map would be given heavy weights, and so the location of the target element would be particularly activated as it is the only one getting activation from both of the heavily weighted feature-salience maps. Hence it is very likely to be the first one sampled irrespective of number of distractors, and search times become independent of distractor number.

Can this class of model explain the pattern of results of the present experiments? The knowledge of the target color could be used to give that particular feature map heavy weighting. Therefore the locations of elements of that color would get a strong signal on the activation map relative to the noncued color. When the target then flashes, less strength of flash would be needed to attract focal attention (and the decision process) to these sites of greater activation. Such an explanation should result in attentional effects for both discrimination and detection experiments; however, we found significant effects only for discrimination tasks, suggesting that focal attention may not be needed (or less of it is required) for the detection tasks. Such a notion of less or no attentional modulation of detection tasks has been previously suggested (see also Found \& Müller, 1996; Nothdurft, 1992; Sagi \& Julesz, 1985), and therefore the changes in this activation map do not influence (or have less influence) on the detection task. This explanation, unlike the others, does not predict that reaction times should alter as set size is altered and so is also consistent with this portion of the data. The notion that the effects of the color cue occur in guiding a search mechanism that allows further processing (so-called focal attention)-rather than in altering an early perceptual representation or decision-level processing - is in line with recent suggestions from other paradigms (Shih \& Sperling, 1996).

\section{Bias in Response?}

It has been argued (e.g., Posner et al., 1980) that the effects of precuing might result from a bias to respond more readily to the cued area rather than to any differences in processing. This would manifest itself as a change in criterion ( $\beta$ in signal detection terms) rather than in sensitivity ( $d^{\prime}$ in signal detection terms) in the measurement of thresholds, but as a speeded response in the measurement of reaction times (because less evidence is required for the buttonpress). One could therefore make a similar argument about the present results: The subject is more biased to press the button when a target is of the prespecified color; hence the faster reaction times. We would like to counter this argument with three points.

1. In other experiments (Brawn, Snowden, \& Wolfe, 1999), we presented displays very similar to those of the present experiments but at the same time as the luminance increment/decrement, the element could also change color (with a probability of .5); the subjects' task was to name either the initial color of the element that flashed or its finishing color. We found very poor performance for the initial color but good performance for the final color, suggesting that subjects have little knowledge of the color of an individual element unless focal attention is located on it. It is therefore hard to see how subjects could be biased if they have little or no knowledge of the color of the element that flashed.

2. Bias effects seem most worrisome in simple reaction time experiments or when the cue gives some indication of the response to be made (see, e.g., Spence \& Driver, 1994). However, the only conditions where we have found effects from precuing are the ones using choice reaction times where the cue gave no indication of the response to be made (i.e., the cue indicated the color of the element but not whether it would darken or lighten).

3. Changes in bias should manifest themselves not only in reaction time speed but also in error rates (false positives for detection experiments; incorrect responses for discrimination experiments). However, we found no sign of such change.

Thus it seems very unlikely that bias can explain the effects demonstrated here. 


\section{Location Uncertainty?}

We have presented our experiments in the context of the paradigm of spatial cuing experiments where knowledge of the location of the target facilitates performance (e.g., Posner et al., 1980). In the present experiments, knowledge of the target color was able to modulate performance in an analogous manner (at least for discrimination tasks). Improvement in performance on some spatial cuing tasks has often been attributed to a reduction in positional uncertainty (e.g., Cohn \& Lashley, 1974; Lappin \& Uttal, 1976; Shaw, 1984). Under conditions where the subject is certain of the target's location, only this location need be "monitored." Under conditions where there is low location certainty, many locations have to be monitored, which leads to a high level of noise (since noise occurs at all locations). Such an analysis has been developed for several tasks, such as searching for a target defined by a difference in brightness from the distractors, and appears to predict the results of such experiments with a high degree of accuracy (Palmer, Ames, \& Lindsey, 1993). One could develop a similar argument for the present experiments. In the color-known conditions, the subject needs to monitor only half the elements, and hence has less noise in which to detect the signal, which results in better performance. However, this line of reasoning is not supported by other aspects of the present data. First, this argument should apply equally to both detection and discrimination tasks, and so comparable effects would be predicted, but this was not the case. Second, this analysis predicts that reaction times should increase as the number of elements (and therefore distractors) increases. This was not the case in the present experiments, and so an explanation based on increased noise due a difference in the number of monitored elements also seems untenable.

\section{Task Difficulty?}

One possible explanation of the difference in the color cuing between detection and discrimination tasks might lie in the differences in reaction time between the tasks. The detection tasks typically had reaction times in the region of 400-500 msec, whereas the discrimination tasks typically had reaction times in the region of $600-800 \mathrm{msec}$. One might argue that the discrimination task is harder than the detection task; hence the subject might pay more attention (or follow the cues with greater vigor) under these more demanding conditions. Against this we found that making the detection task more difficult by decreasing flash strength did not produce a color cuing effect (though, again, the trend in each experiment was toward such an effect). Further, if it were the case that subjects were simply paying more attention, or following the cue with greater vigor, for the discrimination task we should see this reflected in all the measures taken during this task. However, an analysis of the miss rates conducted during the discrimination task (a measure of whether the target was detected rather than discriminated) showed the same pattern of results as the detection task rather than the discrimination task. These results argue against task difficulty per se as an explanation of the difference between the two tasks. In a series of experiments using a somewhat different stimulus, we have examined this issue in much greater detail-comparing reaction time and signal detection measures (including bias-free forced-choice methodology), as well as manipulating task difficulty (Brawn \& Snowden, in press). Accordant with the present findings, these experiments consistently showed greater attentional effects for discrimination tasks relative to detection tasks, but showed no effect of task difficulty.

\section{Electrophysiological Evidence}

Human VEPs to the flashed presentation of a bar stimulus contain several components. Careful analysis of the sources of the these components, combined with sophisticated brain imaging techniques (Clark \& Hillyard, 1996; Heinze et al., 1994), has suggested (1) an early C1 component that is not modulated by changes in attention, (2) a P1 component with slightly longer latency that appears to be generated in lateral extrastriate cortex, and (3) an N1 component with possible parietal and frontal sources. These latter components have been found to be modulated by shifts in spatial attention (Clark \& Hillyard, 1996; Eason, 1981; Luck et al., 1994; Mangun, Hillyard, \& Luck, 1993; Rugg, Milner, Lines, \& Phalp, 1987; van Voorhis \& Hillyard, 1977). For tasks in which the subjects merely had to respond to the presence of the target (detection), the P1 component is modulated by attention, but the N1 component is not. If the subject makes a decision based on the size of the target (discrimination), however, both the $\mathrm{P} 1$ and N1 components are modulated by attention (Mangun \& Hillyard, 1991). Given that in the present experiments we found little or no significant effects of attention to stimulus color on a detection task, but did so for a discrimination task, it is tempting to associate the latter finding with the N1 component. Such a hypothesis could be tested by examination of VEPs under our experimental conditions.

The action of single cells is also modulated by focal attention (see Maunsell, 1995). Motter (1994) has used stimuli similar to those of Experiment 4 while recording from cells in macaque area V4. The animal was given a color cue and was then presented with an array of six items, three of which were one color (let us say red) and three another (green). After some time period, four of the items (two red and two green) were removed and the animal had to judge the orientation of the remaining item in the color of the cue. The crucial recording period was when six items were still visible. The animal will now be paying greater attention to the items that share the same color as the cue. Motter found that many cells (approximately $75 \%$ ) gave a significantly greater response when the element in its receptive field was cued. Taken as a population, the responses were approximately twice as great under color-cued versus not-cued conditions. Thus there appears to be evidence at the single-cell level that attention can be selectively applied to all items of one color but not to spatially interspersed items of a different color. It is 
also of interest to note the time course of this attentional effect. Comparison of cued with noncolor items showed no difference over the first $30 \mathrm{msec}$ or so of the trial, and after that the difference grew gradually and was maximal from around $500 \mathrm{msec}$ and remained so until the trial was terminated (after approximately $1,500 \mathrm{msec}$ ). Hence most experiments in which items are presented for immediate response (as in visual search paradigms) may fail to yield this effect because of its relatively long temporal dynamics.

\section{Conclusion}

In a display that contains many spatially dispersed elements, we have shown that subjects can differentially process one set of these elements based on a common color at least under some circumstances. It would be of interest to see if other stimulus attributes such as common motion, shape, depth, and so forth, also allow for similar effects.

\section{REFERENCES}

ANstIs, S. M. (1974). A chart demonstrating variations in acuity with retinal position. Vision Research, 14, 589-592.

BASHINSKI, H. S., \& BACHARACH, V. R. (1980). Enhancement of perceptual sensitivity as the result of selectively attending to spatial locations. Perception \& Psychophysics, 28, 241-248.

BAYLIS, G. C., \& DRIVER, J. (1992). Visual parsing and response competition: The effect of grouping factors. Perception \& Psychophysics, 51, 145-162.

BeCKLen, R., \& Cervone, D. (1983). Selective looking and the noticing of unexpected events. Memory \& Cognition, 11, 601-608.

BonNel, A.-M., Stein, J.-F., \& Bertucci, P. (1992). Does attention modulate the perception of luminance changes? Quarterly Journal of Experimental Psychology, 44A, 601-626.

Bravo, M. J., \& NAKAYAMA, K. (1992). The role of attention in different visual-search tasks. Perception \& Psychophysics, 51, 465-472.

BRAWN, P. T., \& SNOWDEN, R. J. (1996). The role of attention in the detection of luminance changes: Endogenous versus exogenous cuing. Perception, 25, 3-4S.

BRAwn, P. T., \& SNOWDEN, R. J. (in press). Attention to overlapping objects: Detection and discrimination of luminance changes. Journal of Experimental Psychology: Human Perception \& Performance.

BRaWN,, P. T., SNOWden, R. J., \& Wolfe, J. M. (1999). The minimal conditions for "change blindness": What is hides what was [Abstract] Investigative Ophthalmology \& Visual Science, 40, S49.

Broadbent, D. E. (1958). Perception and communication. London: Pergamon.

Bundesen, C. (1990). A theory of visual attention. Psychological Review, 97, 523-547.

CARTER, R. C. (1982). Visual search with color. Journal of Experimental Psychology: Human Perception \& Performance, 8, 127-136.

CAve, K. R., \& PAShler, H. (1995). Visual selection mediated by location: Selecting successive visual objects. Perception \& Psychophysics, 57, 421-432.

Clark, V. P., \& Hillyard, S. A. (1996). Spatial selective attention affects early extrastriate but not striate components of the visual evoked potential. Journal of Cognitive Neuroscience, 8, 387-402.

COHN, T. E., \& LASHLEY, D. J. (1974). Detectability of a luminance increment: Effect of spatial uncertainty. Journal of the Optical Society of America, 64, 1715-1719.

DownING, C. J. (1988). Expectancy and visual spatial-attention: Effects on perceptual quality. Journal of Experimental Psychology: Human Perception \& Performance, 14, 188-202.

EASON, R. G. (1981). Visual evoked potential correlates of early neural filtering during selective attention. Bulletin of the Psychonomic Society, 18, 203-206.

EGETH, H. E., VirZI, R. A., \& GARBART, H. (1984). Searching for con- junctively defined targets. Journal of Experimental Psychology: Human Perception \& Performance, 10, 32-39.

ERIKSEN, B. A., \& ERIKSEN, C. W. (1974). Effects of noise letters upon the identification of a target letter in a nonsearch task. Perception \& Psychophysics, 16, 143-149.

ERIKSEN, C. W., \& HoffMan, J. E. (1972). Temporal and spatial characteristics of selective encoding from visual displays. Perception $\&$ Psychophysics, 12, 201-204.

FounD, A., \& MǘlLER, H. J. (1996). Searching for unknown feature targets on more than one dimension: Investigating a "dimensionweighting" account. Perception \& Psychophysics, 58, 88-101.

FriedMaN-HiLl, S., \& WolfE, J. M. (1995). Second-order parallel processing: Visual search for the odd item in a subset. Journal of Experimental Psychology: Human Perception \& Performance, 21, 531-551.

GoldsteIN, E. B., \& Fink, S. I. (1981). Selective attention in vision: Recognition memory for superimposed line drawings. Journal of Experimental Psychology: Human Perception \& Performance, 7, 954967.

Green, B. F., \& Anderson, L. K. (1956). Color coding in a visual search task. Journal of Experimental Psychology, 51, 19-24.

Hawkins, H. L., Hillyard, S. A., Luck, S. J., Mouloua, M., DownING, C. J., \& WoodwaRD, D. P. (1990). Visual attention modulates signal detectability. Journal of Experimental Psychology: Human Perception \& Performance, 16, 801-811.

Hawkins, H. L., Shafto, M. G., \& Richardson, K. (1988). Effects of target luminance and cue validity in the latency of visual detection. Perception \& Psychophysics, 44, 484-492.

Heinze, H. J., Mangun, G. R., Burchert, W., Hinrichs, H., Sholz, M., Münte, T. F., Gös, A., Sherg, M., Johannes, S., HunDEShagen, H., Gazzinga, M. S., \& Hillyard, S. A. (1994). Combined spatial and temporal imaging of brain activity during visual selective attention in humans. Nature, 372, 543-546.

Hughes, H. C. (1984). Effects of flash luminance and positional expectancies on visual response latency. Perception \& Psychophysics, 36, 177-184.

Kaptein, N. A., Theeuwes, J., \& van der HeiJden, A. H. C. (1995). Search for a conjunctively defined target can be selectively limited to a color-defined subset of elements. Journal of Experimental Psychology: Human Perception \& Performance, 21, 1053-1069.

Kinchla, R. A. (1992). Attention. Annual Review of Psychology, 43, 711-742.

Kinchla, R. A., Chen, Z., \& Evert, D. (1995). Precue effects in visual search: Data or resource limited? Perception \& Psychophysics, 57, 441-450.

LAARNI, J., Koski, M., \& Nyman, G. (1996). Efficiency of selective attention: Selection by color and location compared. Perception, 25, 1401-1418.

LAmbert, A., \& Hockey, R. (1986). Selective attention and performance with a multidimensional visual display. Journal of Experimental Psychology: Human Perception \& Performance, 12, 484-495.

LAPPIN, J. S.. \& UTTAL, W. R. (1976). Does prior knowledge facilitate the detection of visual targets in random noise? Perception \& Psychophysics, 20, 367-374.

Luck, S. J., Hillyard, S. A., Mouloua, M., WoldorfF, M. G., Clark, V. P., \& Hawkins, H. L. (1994). Effects of spatial cueing on luminance detectability: Psychophysical and electrophysiological evidence for early selection. Journal of Experimental Psychology: Human Perception \& Performance, 20, 887-904.

MANGUN, G. R., \& Hillyard, S. A. (1991). Modulations of sensoryevoked brain potentials indicate changes in perceptual processes during visual-spatial priming. Journal of Experimental Psychology: Human Perception \& Performance, 17, 1057-1074.

Mangun, G. R., Hullyard, S. A., \& LuCK, S. J. (1993). Electrocortical substrates of visual selective attention. In D. Meyer \& S. Kornblum (Eds.), Attention and performance XIV: Synergies in experimental psychology, artificial intelligence, and cognitive neuroscience (pp. 219-243). Cambridge, MA: MIT Press.

MAUNSELL, J. H. R. (1995). The brain's visual world-Representation of visual targets in cerebral cortex. Science, 270, 764-769.

MotTER, B. C. (1994). Neural correlates of attentive selection for color 
or luminance in extrastriate area V4. Journal of Neuroscience, 14, 2178-2189.

Müller, H. J., \& HuMPhreys, G. W. (1991). Luminance increment detection: Capacity limited or not? Journal of Experimental Psychology: Human Perception \& Performance, 17, 107-124.

NaKayama, K., \& Silverman, G. H. (1986). Serial and parallel processing of visual feature conjunctions. Nature, 320, 264-265.

NeISSER, U., \& BECKLEN, R. (1975). Selective looking: Attending to visually specified events. Cognitive Psychology, 7, 480-494.

NoTHDURFT, H.-C. (1992). Feature analysis and the role of similarity in preattentive vision. Perception \& Psychophysics, 52, 355-375.

PaLmer, J. (1994). Set-size effects in visual search: The effect of attention is independent of the stimulus for simple tasks. Vision Research, 34, 1703-1721.

PAlmer, J., AMEs, C. T., \& Lindsey, D. T. (1993). Measuring the effect of attention on simple visual search. Journal of Experimental Psychology: Human Perception \& Performance, 19, 108-130.

POSNER, M. I., \& SNYDER, C. R. (1978). Facilitation and inhibition in the processing of signals. In P. M. A. Rabbit \& S. Dornic (Eds.), Attention and performance V (pp. 669-682). New York: Academic Press.

Posner, M. I., SNyder, C. R., \& Davidson, B. J. (1980). Attention and the detection of signals. Journal of Experimental Psychology: General, 109, 160-174.

PYLYSHYN, Z. W., \& STORM, R. W. (1988). Tracking multiple independent targets: Evidence for a parallel tracking mechanism. Spatial $V i-$ sion, 3, 179-197.

ReINITZ, M. T. (1990). Effects of spatially directed attention on visual encoding. Perception \& Psychophysics, 47, 497-505.

Rock, I., \& GuTMAN, D. (1981). The effect of inattention on form perception. Journal of Experimental Psychology: Human Perception \& Performance, 7, 275-285.

Rugg, M. D., Milner, A. D., Lines, C. R., \& Phalp, R. (1987). Modulation of visual event-related potentials by spatial and non-spatial visual selective attention. Neuropsychologia, 25, 85-96.

SAGI, D. (1988). The combination of spatial frequency and orientation is effortlessly perceived. Perception \& Psychophysics, 43, 601-603.

SAGI, D., \& JULESZ, B. (1985). "Where" and "what" in vision. Science, 228, $1217-1219$.

SHAW, M. L. (1984). Division of attention among spatial locations: A fundamental difference between detection of letters and detection of luminance increments. In H. Bouma \& D. G. Bouwhuis (Eds.), Attention and performance $X$ : Control of language processes (pp. 109-121). Hillsdale, NJ: Erlbaum.
SHIH, S., \& SperLING, G. (1996). Is there feature based attention in visual search? Journal of Experimental Psychology: Human Perception \& Performance, 22, 758-779.

SмIтH, S. L. (1962). Color coding and visual search. Journal of Experimental Psychology, 64, 434-440.

SNowden, R. J. (1998). Texture segregation and visual search: A comparison of the effects of random variations along irrelevant dimensions. Journal of Experimental Psychology: Human Perception \& Performance, 24, 1354-1367.

SNOwden, R. J., \& Hess, R. F. (1992). Temporal frequency filters in the human peripheral visual field. Tision Research, 32, 61-72.

SPENCE, C. J., \& DRIVER, J. (1994). Covert spatial orienting in audition: Exogenous and endogenous mechanisms. Journal of Experimental Psychology: Human Perception \& Performance, 20, 555-574.

STERNBERG, S. (1969). The discovery of processing stages: Extensions of Donder's method. In W. G. Koster (Ed.), Attention and performance $I I$ (pp. 276-315). Amsterdam; North-Holland.

Stoffregen, T. A., \& Becklen, R. C. (1989). Dual attention to dynamically structured naturalistic events. Perceptual \& Motor Skills, 69, 1187-1201.

Treisman, A. (1982). Perceptual grouping and attention in visual search for features and objects. Journal of Experimental Psychology: Human Perception \& Performance, 8, 194-214.

Treisman, A. (1988). Features and objects: The fourteenth Barlett memorial lecture. Quarterly Journal of Experimental Psychology, 40, 201-237.

Treisman, A., \& Gelade, G. (1980). A feature integration theory of attention. Cognitive Psychology, 16, 97-136.

Tsal, Y., \& LAVIE, N. (1988). Attending to color and shape: The special role of location in selective visual processing. Perception \& Psy. chophysics, 44, 15-21.

van der Heijden, A. H. C., Kurvink, A. G., De Lange, L., de Leeuw, F., \& VAN DER GEEST, J. N. (1996). Attending to color with proper fixation. Perception \& Psychophysics, 58, 1224-1237.

VAN VOORHIS, S. T., \& HILLYARD, S. T. (1977). Visual evoked potentials and selective attention to points in space. Perception \& Psychophysics, 22, 54-62.

WolFe, J. M. (1994). Guided Search 2.0: A revised model of visual search. Psychonomic Bulletin \& Review, 1, 202-238.

(Manuscript received June 12, 1997; revision accepted for publication May 26, 1998.) 